\section{Age- and gender- related tear function changes in normal population}

M Ozdemir and $\mathrm{H}$ Temizdemir

\begin{abstract}
Purpose Schirmer and tear film break-up time tests are most commonly used in the diagnosis of dry eye syndrome. The aim of the study is to investigate the age- and gender-related changes in the results of Schirmer and tear film break-up time tests in normal population.

Methods This randomized prospective study included 140 normal volunteers (70 male and 70 female subjects) with no ocular symptoms or ocular surface disorders. Schirmer and tear film break-up time tests were assessed in only one eye (right) of each subject. The study subjects were divided into seven groups according to their ages. Each group was composed of 20 eyes of 20 subjects (10 male and 10 female subjects). For variance analysis, Kruskal-Wallis test was used. Mann-Whitney $U$-test was performed for double comparisons. Results We detected a statistically highly significant difference among the age groups in respect to the tear film break-up time test $(P<0.001)$, especially there was a statistically highly significant difference between the younger and older age groups. There was no statistically significant difference in the Schirmer test results. But, we found that Schirmer test values were gradually decreased with advancing age. Tear function tests did not show statistically significant difference according to sex.

Conclusions Our results reveal that there is a decline in the tear function test values, especially the tear film break-up time test values with advancing age. We suggest that the age of subjects should be taken into consideration in the evaluation of tear function test results.
\end{abstract}

Eye (2010) 24, 79-83; doi:10.1038/eye.2009.21;

published online 20 February 2009

Keywords: age; gender; Schirmer test; tear film break-up time; tear function
Introduction

Currently, dry eye syndrome or

keratoconjunctivitis sicca is seen with gradually increased frequency in especially middle and advanced ages throughout the world. An estimate of tear secretion and tear film stability is regarded as an important aspect of dry eye diagnosis, and for this purpose, the Schirmer and the tear film break-up time (BUT) tests are currently in use. ${ }^{1-4}$ A measurement of $<10 \mathrm{~mm}$ of wetting is commonly accepted as abnormal for the Schirmer test when it is performed with topical anaesthesia and a value of $<10 \mathrm{~s}$ is considered as abnormal for the tear film BUT test. $^{5,6}$ But, it is not known whether the normal values of these tests change with advancing age or gender.

It is known that chronic marginal blepharitis is more frequently encountered with advanced ages especially in men, ${ }^{7,8}$ and the tear secretion declines after menopause in women. ${ }^{9}$ Because of these data, we thought that tear functions may change with the advancing age and gender. But, to our literature search, there is only one report about age-related alterations of the tear function tests. ${ }^{10}$ This report examined only Schirmer test; it did not evaluate the tear film BUT. To our knowledge, there is no report about gender-related changes of tear function in current literature.

In this study, we aimed to investigate the ageand gender-related changes in the results of Schirmer and tear film BUT tests in normal population.

\section{Materials and methods}

This study was conducted by Ophthalmology Department of Kahramanmaras Sutcu Imam University, Faculty of Medicine, between August 2007 and January 2008. The Local Medical Ethics Committee approval for the study was obtained, and all patients included in
Faculty of Medicine, Department of Ophthalmology, Kahramanmaras Sutcu Imam University, Kahramanmaras, Turkey

Correspondence: M Ozdemir, Department of Ophthalmology, Kahramanmaras Sutcu Imam University, Hastane Cad, Kahramanmaras, Turkey 46100, Turkey Tel: + 9034422123 37; Fax: + 903442212371. E-mail: drmozdemir@ hotmail.com

Received: 18 June 2008 Accepted in revised form: 16 January 2009 Published online: 20 February 2009 
the study gave the written informed consent. The study protocol followed the guidelines of the Declaration of Helsinki.

\section{Case selection}

Participants included patients, employees, and students at the Kahramanmaras Sutcu Imam University, Faculty of Medicine, Kahramanmaras, Turkey. Twenty normal subjects were selected for each decade. Any history of ocular surgery or ocular trauma, any ocular surface disorder, current contact lens use, any topical or systemic drug use, any systemic disorder, such as Sjögren syndrome giving rise to dry eye, or cutaneous disorders, such as contact dermatitis or atopic dermatitis, were regarded as exclusion criteria.

\section{Ocular examinations}

All subjects underwent a complete ophthalmic examination including corrected visual acuity, anterior segment examination, and fundus examination. Subjective complaints, such as burning, itching, tearing, foreign body sensation, or photophobia were asked. The Schirmer and the tear film BUT tests were performed in only one eye (right) of each subject.

Tear film BUT was undertaken without the use of any anaesthetics after instilling one drop of fluorescein $2 \%$ (diluted from fluorescite 10\%, Alcon-Couvreur, Puurs, Belgium) into the lower fornix. To encourage the distribution of fluorescein, subjects were instructed to blink several times for a few seconds. The tear film was observed using a blue cobalt filter under wide lighting without catching the eyelids. The interval after the last blinking to the appearance of first black spot was detected. The test is repeated three times, and the median score was recorded in seconds.

After resting period for $20 \mathrm{~min}$, the modified Schirmer basal secretion test was performed in the following manner: one drop of proparacaine hydrochloride $0.5 \%$ (Alcaine, Alcon-Couvreur, Puurs, Belgium) for topical anaesthesia was instilled to the eyes, and the conjunctival fornix was dried with a cotton tip applicator. After a 2-min waiting period, Standard Whatman No. 41 filter paper strip, $5 \mathrm{~mm}$ wide and $35 \mathrm{~mm}$ long (Whatmann, Maidstone, UK), was folded $5 \mathrm{~mm}$ from one end and placed between the lower eyelid and the globe, taking care not to touch the cornea, at the junction between the middle and lateral third of the eyelid. The patient was allowed to blink normally. Wetting was measured at $5 \mathrm{~min}$ in millimetres.

\section{Statistical analysis}

For variance analysis, Kruskal-Wallis test was used. Mann-Whitney $U$-test was performed for the double comparisons for the age and sex groups. A $P$-value of $<0.05$ was accepted as statistically significant.

\section{Results}

Mean ages of the study subjects were presented in Table 1. Each group included 10 male and 10 female subjects. The study groups were similar in respect to mean age and gender distribution.

Mean Schirmer values in both sex groups were presented in Table 2. Although there was no statistically significant difference, the Schirmer values were found to be gradually decreased with advancing age in both sex groups (Figure 1).

In our study, we found a statistically highly significant decline of the tear film BUT test values with advancing age in both sex groups $(P<0.001$; Table 3 , Figure 2$)$. There was a statistically significant difference between the younger and older age groups, such as groups 1 and 7, or groups 2 and 7 in the tear film BUT results (Table 3).

The Schirmer test values were similar in all groups when a comparison was carried out according to sex (Table 2). But, significant differences between male and female subjects were detected in decades $2(P=0.012)$ and $4(P=0.040)$ in respect to the tear film BUT test values, but there was no significant difference between the sex groups in the other decades.

Table 1 Mean \pm SD ages according to groups and sex

\begin{tabular}{lccrr}
\hline & Number of the subjects & Male & Female & Pa \\
\hline Group 1 (decade 2) & 10 & $17.00 \pm 3.19(11-20)$ & $17.8 \pm 1.03(16-19)$ & 0.796 \\
Group 2 (decade 3) & 10 & $23.80 \pm 3.01(21-29)$ & $25.00 \pm 2.10(22-28)$ & 0.353 \\
Group 3 (decade 4) & 10 & $32.20 \pm 1.61(31-35)$ & $33.90 \pm 2.51(31-38)$ & 0.063 \\
Group 4 (decade 5) & 10 & $47.20 \pm 2.29(44-50)$ & $45.10 \pm 2.76(41-48)$ & 0.079 \\
Group 5 (decade 6) & 10 & $54.40 \pm 3.13(51-59)$ & $54.60 \pm 3.13(51-59)$ & 0.971 \\
Group 6 (decade 7) & 10 & $63.60 \pm 2.60(61-68)$ & $62.00 \pm 1.56(60-65)$ & 0.143 \\
Group 7 (decade 8) & 10 & $75.40 \pm 4.42(71-86)$ & $73.50 \pm 1.84(71-75)$ & 0.315 \\
\hline
\end{tabular}

${ }^{\mathrm{a} M a n n-W h i t n e y} U$-test. 
Table 2 Schirmer test values according to age and sex groups

\begin{tabular}{lccr}
\hline & \multicolumn{2}{c}{ Schirmer test values (mean $\pm S D($ range)) } & \multirow{2}{*}{$P^{\mathrm{a}}$} \\
\cline { 2 - 3 } & Male $(\mathrm{n}=70)$ & Female $(\mathrm{n}=70)$ & 0.853 \\
\hline Group 1 (decade 2) & $17.80 \pm 6.61(8-28)$ & $18.40 \pm 7.39(9-35)$ & 0.427 \\
Group 2 (decade 3) & $19.90 \pm 7.35(10-34)$ & $16.80 \pm 8.76(7-33)$ & 0.402 \\
Group 3 (decade 4) & $14.50 \pm 6.22(9-30)$ & $12.90 \pm 7.49(6-32)$ & 0.970 \\
Group 4 (decade 5) & $13.50 \pm 8.60(4-35)$ & $12.40 \pm 7.01(4-24)$ & 0.362 \\
Group 5 (decade 6) & $10.10 \pm 6.08(4-22)$ & $13.00 \pm 5.96(5-24)$ & 0.849 \\
Group 6 (decade 7) & $12.10 \pm 5.08(3-20)$ & $11.00 \pm 5.57(4-21)$ & 0.732 \\
Group 7 (decade 8) & $11.40 \pm 4.85(5-20)$ & 0.060 & \\
$P^{\mathrm{b}}$ & 0.125 & & \\
\hline
\end{tabular}

${ }^{a}$ Mann-Whitney $U$-test.

${ }^{\mathrm{b}}$ Kruskal-Wallis test.

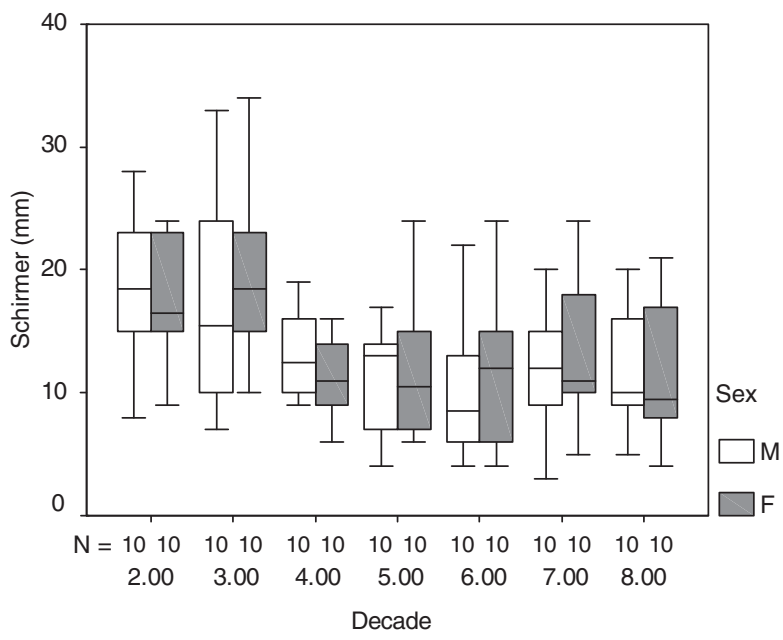

Figure 1 Change of the Schirmer test results according to advancing age in both sex.

\section{Discussion}

Dry eye syndrome is a clinical condition characterised by deficient tear production or excessive tear evaporation. The population-based studies revealed that dry eye syndrome, an important health problem, is seen throughout the world with a frequency of up to $33 \% .^{11}$ The Schirmer and tear film BUT tests are most commonly used tests in the diagnosis of dry eye. The basal Schirmer test quantitatively evaluates the basic tear production. The tear film BUT test reflects tear stability and composition.

It is known that the comprehension of normal values of the tests used in the diagnosis of any disease is essential in making an accurate diagnosis. The diagnostic tests may have diverse normal values in different ages and sexes. To our knowledge, it is not well known how the normal values of the Schirmer and the tear film BUT tests change according to age and gender. Because of that, we aimed to investigate the age- and gender-related changes in the results of Schirmer and tear film BUT tests in normal population.

In this study, we found that the tear film BUT values decreased significantly with aging. Age-related reduction of the tear film BUT test values was parallel in both genders. These data show that the stability of the tear film is gradually reduced with advancing age. There may be several causes of this condition. With advancing age, decreased lid laxity and disorder of the dynamics of eyelid closure may cause a failure of distribution of the tear film. Earlier studies detected that disorder of the lid margin morphology and decreased meibomian gland secretion are very common findings among the elderly patients. These problems lead to the increase of surface tension and early evaporation of tear film. ${ }^{7,8,12,13}$ Some studies introduced a relationship between the serum androgen level and meibomian gland function. ${ }^{14-16}$ According to these studies, decreased serum androgen level may cause a disorder of meibomian gland functions and evaporative dry eye. A mucin deficiency may occur and this may cause tear instability in advancing age, but we cannot found any study in the literature about this possibility.

Despite the Schirmer test results of our study were found to be insignificant statistically, they were determined to be decreased parallelly in both sexes with advancing age. Mathers $e t a l^{10}$ showed that the tear production decreased inversely proportionally to the age in their study performed with fluorophotometry and Schirmer test without anaesthesia. Hirase $e t \mathrm{al}^{21}$ in their study performed with fluorophotometry, found a statistically significant difference in the tear volume, tear turnover rate, and basic tear flow rate between younger and older subjects in favour of the young. It remains unclear as to why the tear production diminishes with the advancing age. However, it is known that in women reduced sex hormone levels after menopause cause dryness in all mucosal tissues as well as affecting the tear 
Table 3 Tear film BUT test values according to age groups

\begin{tabular}{|c|c|c|c|}
\hline & \multicolumn{2}{|c|}{ Tear Film BUT test values (mean \pm SD (range)) } & \multirow[t]{2}{*}{$P^{\mathrm{a}}$} \\
\hline & Male $(n=70)$ & Female $(n=70)$ & \\
\hline Group 1 (decade 2) & $44.10 \pm 6.15(36-52)$ & $34.90 \pm 6.82(25-49)$ & 0.012 \\
\hline Group 2 (decade 3) & $35.20 \pm 7.95(26-50)$ & $36.30 \pm 7.39(20-46)$ & 0.495 \\
\hline Group 3 (decade 4) & $26.80 \pm 5.09(17-40)$ & $34.00 \pm 5.20(28-42)$ & 0.040 \\
\hline Group 4 (decade 5) & $25.80 \pm 7.11(14-33)$ & $27.00 \pm 6.77(17-38)$ & 0.867 \\
\hline Group 5 (decade 6) & $22.10 \pm 8.98(11-28)$ & $24.60 \pm 6.46(12-38)$ & 0.379 \\
\hline Group 6 (decade 7) & $18.40 \pm 5.23(11-29)$ & $16.40 \pm 3.17(11-26)$ & 0.595 \\
\hline Group 7 (decade 8) & $13.10 \pm 3.18(9-18)$ & $15.90 \pm 4.35(11-23)$ & 0.134 \\
\hline$P^{\mathrm{b}}$ & $<0.001$ & $<0.001$ & \\
\hline
\end{tabular}

${ }^{a}$ Mann-Whitney $U$-test.

${ }^{\mathrm{b}}$ Kruskal-Wallis test.

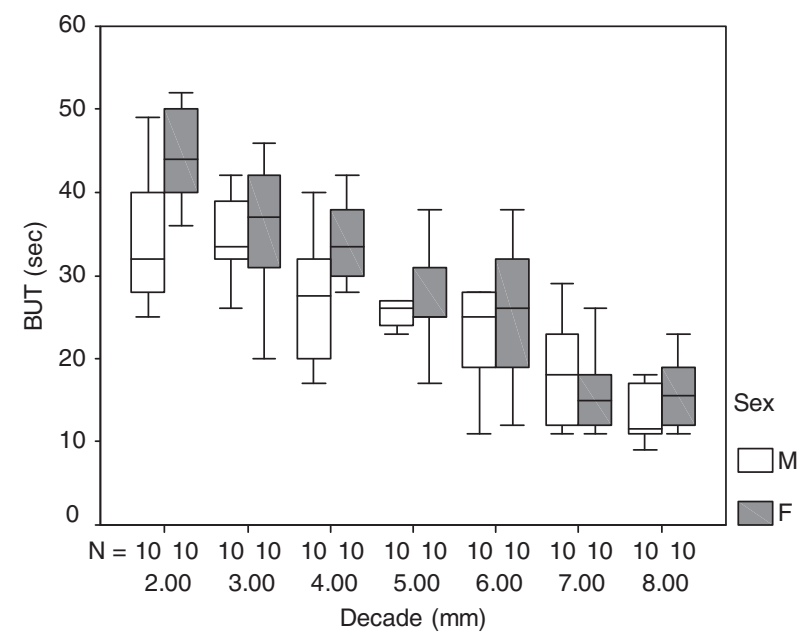

Figure 2 Change of tear film BUT test results according to advancing age in both sexes.

production negatively. ${ }^{10}$ Similarly, sex hormones in men also play an important role in the regulation of tear production. Decreasing androgen levels with the advancing age may lead to the reduction of tear production. ${ }^{14-16}$ The demonstration of androgen treatment to increase tear production in mice with Sjogren syndrome also substantiates this idea. ${ }^{22}$

When the Schirmer test results were compared separately for every decades, it was seen that there was no significant difference between men and women. The results of tear film BUT test were found higher in men in the second decade and in women in the fourth decade. No men-women difference was found in other decades. This issue was not considered to be significant and thought to be relevant with the low number of subjects in the groups. In our study, the tear film BUT test values in younger age groups were much higher than in earlier reports. ${ }^{17-20}$ We think that this condition might be caused by the racial disparity because many of these reports were performed on different races.

In conclusion, our study showed that normal values of tear function tests change with the advancing age, especially tear film BUT decreases markedly. Besides, the change in tear functions is parallel in both sexes and shows no marked difference. We suggest that the age of patients should also be taken into consideration when the results of Schirmer and BUT tests are evaluated in the diagnosis and follow-up of dry eye syndrome.

\section{References}

1 Farris FL, Gilbard JP, Stuchell RN, Mandell ID. Diagnostic tests in keratoconjunctivitis sicca. CLAO J 1983; 9: 23-28.

2 Goren MB, Goren SB. Diagnostic tests in patients with symptoms of keratoconjunctivitis sicca. Am J Ophthalmol 1988; 106: 570-574.

3 Lemp MA. Report of the national eye institute/industry workshop on clinical trials in dry eyes. CLAO J 1995; 21: 221-232.

4 Schirmer O. Studien zur Physiologie und Pathologie der Träneabsonderung und Tränenabfuhr. Graefes Arch Klin Exp Ophthalmol 1903; 56: 197-291.

5 Jones LT. The lacrimal secretory system and its treatment. Am J Ophthalmol 1966; 62: 47-60.

6 Kanski JJ. Clinical Ophthalmology, 3rd edn. Betterworth Heinemann: London, 1994.

7 Hykin PG, Bron AJ. Age-related morphological changes in lid margin and meibomian gland anatomy. Cornea 1992; 11: 334-342.

8 Den S, Shimizu K, Ikeda T, Tsubota K, Shimmura S, Shimazaki J. Association between meibomian gland changes and aging, sex, or tear function. Cornea 2006; 25: 651-655.

9 Mathers WD, Stovall D, Lane JA, Zimmerman MB, Johnson $S$. Menopause and tear function: the influence of prolactin and sex hormones on human tear production. Cornea 1998; 17: 353-358.

10 Mathers WD, Lane JA, Zimmerman MB. Tear film changes associated with normal aging. Cornea 1996; 15: 229-234. 
11 Shimmura S, Shimazaki J, Tsubota K. Results of a population-based questionnaire on the symptoms and lifestyles associated with dry eye. Cornea 1999; 18: 408-411.

12 Patel S, Wallace I. Tear meniscus height, lower punctum lacrimale, and the tear lipid layer in normal aging. Optom Vis Sci 2006; 83: 731-739.

13 Uchino M, Dogru M, Yagi Y, Goto E, Tomita M, Kon T et al. The features of dry eye disease in a Japanese elderly population. Optom Vis Sci 2006; 83: 797-802.

14 Sullivan DA, Krenzer KL, Sullivan BD, Tolls DB, Toda I, Dana MR. Does androgen insufficiency cause lacrimal gland inflammation and aqueous tear deficiency? Invest Ophthalmol Vis Sci 1999; 40: 1261-1265.

15 Sullivan DA, Sullivan BD, Evans JE, Schirra F, Yamagami H, Liu $\mathrm{M}$ et al. Androgen deficiency, meibomian gland dysfunction, and evaporative dry eye. Ann NY Acad Sci 2002; 966: 211-222.
16 Sullivan DA. Androgen deficiency \& dry eye syndromes. Arch Soc Esp Oftalmol 2004; 79: 49-50.

17 Maudgil SS, Khurana AK, Singh M, Parmar IP, Ahluwalia BK. Tear film flow and stability in normal Indian subjects. Indian J Ophthalmol 1989; 37: 182-183.

18 Cho P, Yap M. Age, gender, and tear break-up time. Optom Vis Sci 1993; 70: 828-831.

19 Mohidin N, Bay TC, Yap M. Non-invasive tear break-up time in normal Malays. Clin Exp Optom 2002; 85: 37-41.

20 Emina MO, Emina B. A study on tear stability, tear meniscus height and cornea curvature on healthy black subjects. S Afr Optom 2006; 65: 107-111.

21 Hirase K, Shimizu A, Yokoi N, Nishida K, Kinoshita S. Agerelated alteration of tear dynamics in normal volunteers. Nippon Ganka Gakkai Zasshi 1994; 98: 575-578.

22 Sullivan DA, Edwards JA. Androgen stimulation of lacrimal gland function in mouse models of Sjögren's syndrome. J Steroid Biochem Mol Biol 1997; 60: 237-245. 